\title{
Knockdown of NUPR1 inhibits the growth of U266 and RPMI8226 multiple myeloma cell lines via activating PTEN and caspase activation-dependent apoptosis
}

\author{
CHENSI ZENG ${ }^{1}$, XINGXIN LI $^{1}$, ANMAO LI $^{1}$, BEI YI $^{1}$, XI PENG $^{1}$, XI HUANG $^{2}$ and JIANBIN CHEN ${ }^{1}$ \\ ${ }^{1}$ Department of Hematology, The First Affiliated Hospital of Chongqing Medical University, Chongqing 400016; \\ ${ }^{2}$ Department of Hematology, Hechuan Hospital of The First Affiliated Hospital of Chongqing Medical University, \\ Chongqing 401520, P.R. China
}

Received December 28, 2017; Accepted June 15, 2018

DOI: $10.3892 / o r .2018 .6544$

\begin{abstract}
Nuclear protein-1 (NUPR1) is a stress response factor that is important in the development of several human malignant tumor cells. However, the role of NUPR1 in multiple myeloma (MM) remains to be fully elucidated. In the present study, it was found that the mRNA levels of NUPR1 were significantly higher in specimens from patients with $\mathrm{MM}$ and MM cell lines (U266 and RPMI8226) than in cells of normal human bone marrow. The present study was undertaken to investigate the function of NUPR1 in the growth and apoptosis of MM cell lines. A lentivirus-mediated short hairpin RNA was used to specifically inhibit the mRNA and protein expression of NUPR1 in the U266 and RPMI8226 MM cell lines. Flow cytometry and Cell Counting Kit- 8 assays were applied to examine the apoptosis and proliferation of U266 and RPMI8226 cell lines. The results revealed the inhibitory effect of NUPR1 silencing on the proliferation of U266 and RPMI8226 cells through inducing apoptosis, and arrest of cell cycle at the $\mathrm{G}_{0} / \mathrm{G}_{1}$ phase. Furthermore, NUPR 1 silencing caused activation of caspase- $-3,-8$ and -9 and influenced specific gene expression, including an increase of phosphatase and tensin homolog (PTEN) and decrease of B-cell lymphoma 2 and proliferating cell nuclear antigen. These findings showed that NUPR1 may be involved in the proliferation and apoptosis of MM cells by adjusting caspase proteins and PTEN, suggesting that NUPR1 may be a novel therapeutic target for MM.
\end{abstract}

Correspondence to: Dr Jianbin Chen, Department of Hematology, The First Affiliated Hospital of Chongqing Medical University, 1 Youyi Road, Yuzhong, Chongqing 400016, P.R. China

E-mail:605178418@qq.com

Key words: multiple myeloma, nuclear protein-1, short hairpin RNA, apoptosis, leukemia

\section{Introduction}

Multiple myeloma (MM) is an incurable disease of hematological malignancies, characterized by the abnormal proliferation of plasma cells and overexpression of monoclonal immunoglobulin, which accounts for $\sim 10 \%$ of hematopoietic neoplasias with increasing incidence worldwide. MM can be divided into hyperdiploid multiple myeloma (HD-MM) and non-HD-MM subtypes by fluorescence in situ hybridization (1). The majority of cases of MM are from the original premalignant state, known as monoclonal gammopathy of undetermined significance, to smoldering MM, truly overt and symptomatic MM, and finally extramedullary $\mathrm{MM}$ /plasma cell leukemia $(2,3)$. Despite agents, including proteasome inhibitors and immunomodulatory drugs, and rapidly developing stem cell transplantation technology, which have significantly improved the efficacy of treatment and prognosis of patients with MM, treatment for relapse remains limited.

Nuclear protein-1 (NUPR1), also known as p8 and candidate of metastasis 1 , was first described in pancreatic acinar cells of rats when evaluating the molecular changes in the injured pancreas (4). The NUPR1 gene is located on human chromosome 16p11.2. The length of its cDNA is $719 \mathrm{bp}$, and its open reading frame is $249 \mathrm{bp}$, encoding a protein with a molecular weight of 8,872.7 Da (5). Studies have found that Nupr1 has a wide regulatory role in cell proliferation, migration and apoptosis, and is involved in the development of various types of tumor, including pancreatic cancer, liver cancer, bladder cancer and breast cancer (6-9). It has been shown that NUPR1 also regulates cell sensitivity to drugs in hepatocarcinoma cells (7). It has been reported that there is a specific NUPR1 expression profile in HD-MM, which may become a novel drug target (10). However, the characterization of its effects on MM cells remains to be fully elucidated.

In the present study, the effect of NUPR1 in MM cells was examined; it was found that NUPR1 was upregulated in MM cell lines (U266 and RPMI8226), and NUPR1 was then silenced using lentiviral short hairpin (sh)RNA in the U266 and RPMI8226 cells. The functional effect of NUPR1 was examined by proliferation, apoptosis and cell cycle assays. In order to examine the possible mechanism underlying these 
effects, the expression of genes known to be associated with proliferation and apoptosis were examined by reverse transcription-quantitative polymerase chain reaction (RT-qPCR) and western blot analyses.

\section{Materials and methods}

Cell culture and sample collection. The U266 and RPMI8226 human MM cell lines were donated by Professor Jian Hou at The Second Military Medical University (Shanghai, China). The U266 and RPMI8226 cells were cultured in RPMI-1640 medium (Gibco; Thermo Fisher Scientific, Inc., Waltham, MA, USA), supplemented with $10 \%$ heat-inactivated fetal bovine serum (FBS; Gibco; Thermo Fisher Scientific, Inc.) at $37^{\circ} \mathrm{C}$ in a humidified incubator with $5 \% \mathrm{CO}_{2}$. Bone marrow specimens were obtained from four patients with MM (two females and two males aged 77, 63, 75 and 61 years), and four healthy individuals (one female and three males aged 61, 65, 53 and 55 years) admitted to The First Affiliated Hospital of Chongqing Medical University (Chongqing, China). These specimens were collected between April 2016 and October 2016. The diagnosis was established according to standard morphological and immunophenotypic criteria and revised international staging system (11). All specimens were obtained in accordance with the Research Ethics Board of the First Affiliated Hospital of Chongqing Medical University.

Lentiviral vector construction and cell infection. The lentiviral shRNAs were designed and synthesized by Shanghai GeneChem Co., Ltd. (Shanghai, China). The RNA interference (RNAi) target sequence for NUPR1 was 5'-CCAAGCTG CAGAATTCAGA-3' whereas the control non-silencing RNA sequence was 5'-TTCTCCGAACGTGTCACGT-3'. The lentivirus was produced in 293T cells (American Type Culture Collection, Manassas, VA, USA) as discussed previously (12). The U266 and RPMI8226 cells in the exponential phase were plated in 24 -well plates $\left(5 \times 10^{4}\right.$ cells/well), infected with NUPR1-shRNA lentivirus or NC-shRNA lentivirus in the presence of $1 \mu \mathrm{g} / \mathrm{ml}$ polybrene at a set multiplicity of infection of 100 . After 5 days, the transfection efficiency was estimated by flow cytometry.

$R N A$ extraction and $R T-q P C R$ analysis. Total RNA was extracted from cells with TRIzol reagent (Invitrogen; Thermo Fisher Scientific,Inc.) according to the manufacturer's protocol. The RT reaction was performed using the Prime Script ${ }^{\mathrm{TM}}$ RT reagent kit according to the manufacturer's protocol (Takara Biotechnology Co., Ltd., Dalian, China). cDNA was reverse transcripted from 1,000 ng total RNA RT-qPCR analysis was performed using a CFX96 Real-Time PCR system (Bio-Rad Laboratories, Inc., Hercules, CA, USA) and a SYBR Premix Ex Taq ${ }^{\mathrm{TM}}$ II PCR kit (Takara Biotechnology Co., Ltd.). The primer pairs were as follows: NUPR1, forward 5'-AGGACT TATTCCCGCTGACTGA-3' and reverse 5'-TGCCGTGCG TGTCTATTTATTG-3'; B-cell lymphoma 2 (Bcl-2), forward 5'-AACATCGCCCTGTGGATGAC-3' and reverse 5'-GAC TTCACTTGTGGCCCAGAT-3'; proliferating cell nuclear antigen (PCNA), forward 5'-CTCGTCCCACGTCTCTTT GG-3' and reverse 5'-CGCGTTATCTTCGGCCCTTA-3'; phosphatase and tensin homolog (PTEN), forward 5'-ACA

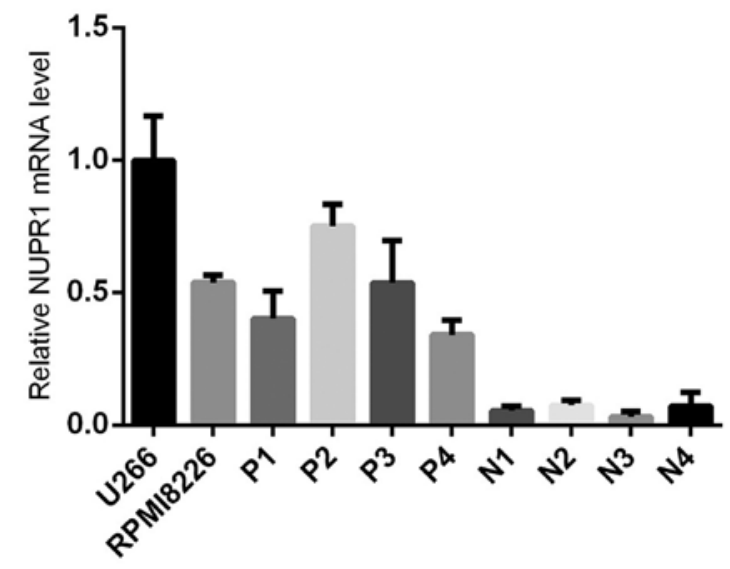

Figure 1. Expression of NUPR1 in MM cell lines and samples. Reverse transcription-quantitative polymerase chain reaction analysis showed increased expression of NUPR1 in the U266 and RPMI8226 MM cell lines and specimens of patients, compared with the cells of normal bone marrow. P1-P4 are specimens of patients with MM; N1-N4 are specimens from healthy human bone marrow. MM, multiple myeloma; NUPR1, nuclear protein-1.

CGACGGGAAGACAAGTT-3' and reverse 5'-CTGGTCCTG GTATGAAGAATG-3'; $\beta$-actin, used as an internal control, forward 5'-CCACGAAACTACCTTCAACTCC-3' and reverse 5'-GTGATCTCCTTCTGCATCCTGT-3'. The PCR reactions consisted of $30 \mathrm{sec}$ at $95^{\circ} \mathrm{C}$, followed by 40 cycles at $95^{\circ} \mathrm{C}$ for $5 \mathrm{sec}$ and annealing at $60^{\circ} \mathrm{C}$ for $30 \mathrm{sec}$. Relative gene expression was calculated using the $2^{-\Delta \Delta \mathrm{Cq}}$ method (13).

Western blot analysis. Total protein was extracted from the MM cells, and protein quantification was performed using the BCA method (Beyotime Institute of Biotechnology, Haimen, China). Subsequently, equal quantities of protein $(40 \mu \mathrm{g})$ were separated by SDS-PAGE (12\%), transferred onto PVDF membranes, and then blocked with 5\% non-fat milk. The membranes and corresponding specific antibodies were incubated at $4^{\circ} \mathrm{C}$ overnight. The following antibodies were used as primary antibodies: Rabbit anti-human NUPR1 antibody (1:1,000; cat. no. MBS420484; Novus Biologicals LLC, Littleton, CO, USA); PCNA (1:1,000; cat. no. ab18197; Abcam, Cambridge, UK) and PTEN (1:1,000; cat. no. ab31392; Abcam); cleaved caspase-3 (1:1,000; cat. no. Asp175; Cell Signaling Technology, Inc., Danvers, MA, USA,), cleaved caspase-8 (1:1,000; cat. no. Asp391; Cell Signaling Technology), cleaved caspase-9 (1:1,000; cat. no. Asp315; Cell Signaling Technology); Bcl-2 (1:800; cat. no. 12789-1-AP; ProteinTech Group, Inc., Wuhan, China); GAPDH (1:5,000; cat. no. 10494-1-AP; ProteinTech Group). These membranes were incubated with secondary antibodies $(1: 3,000$; cat. no. 7074; Cell Signaling Technology) for $2 \mathrm{~h}$ at $37^{\circ} \mathrm{C}$, following washing with TBS-Tween-20 the following day. Excess antibody was removed with TBS-Tween-20 prior to incubation in ECL. The western blot assays were repeated three times. The band intensity was analyzed using Quantity One software (version 4.5.0; Bio-Rad Laboratories) according to the manufacturer's instructions.

Cell Counting Kit-8 (CCK-8) assay for cell proliferation. The proliferation of the U266 and RPMI8226 cells was 


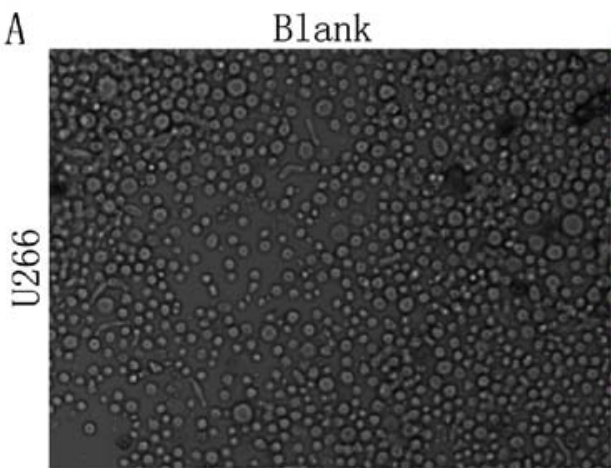

Blank
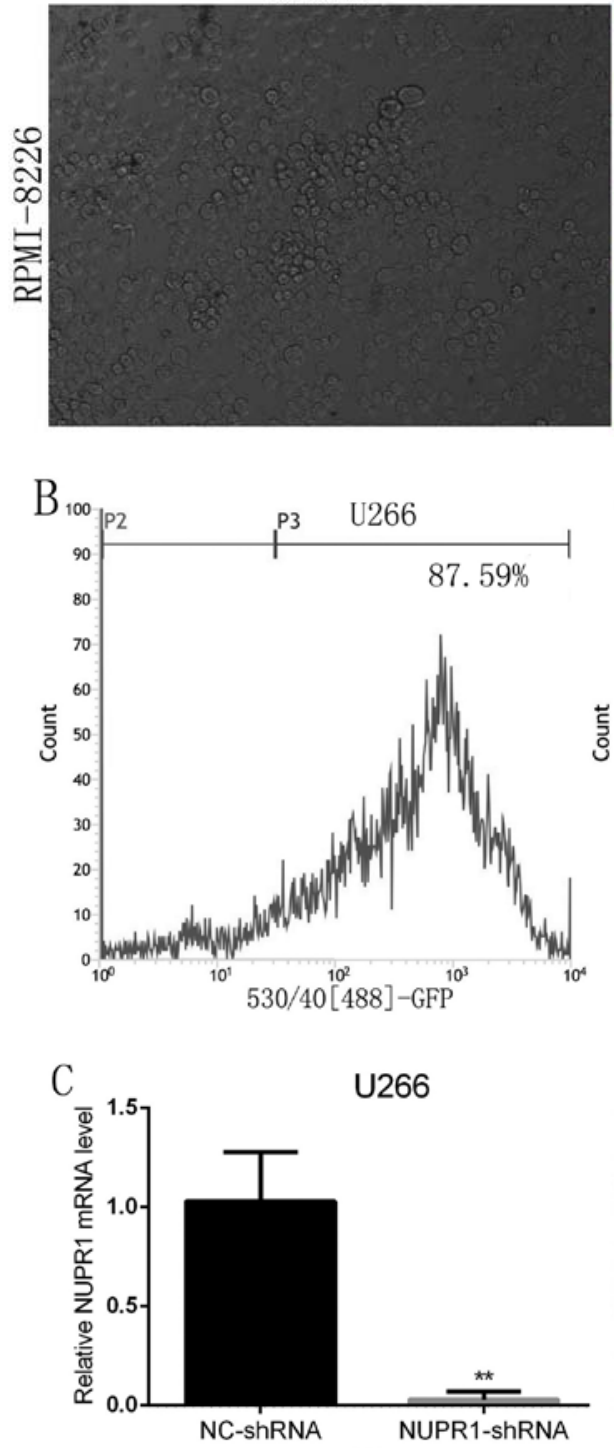

U266

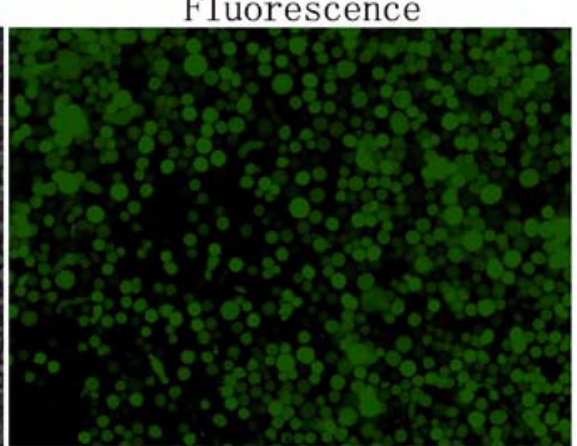

Fluorescence
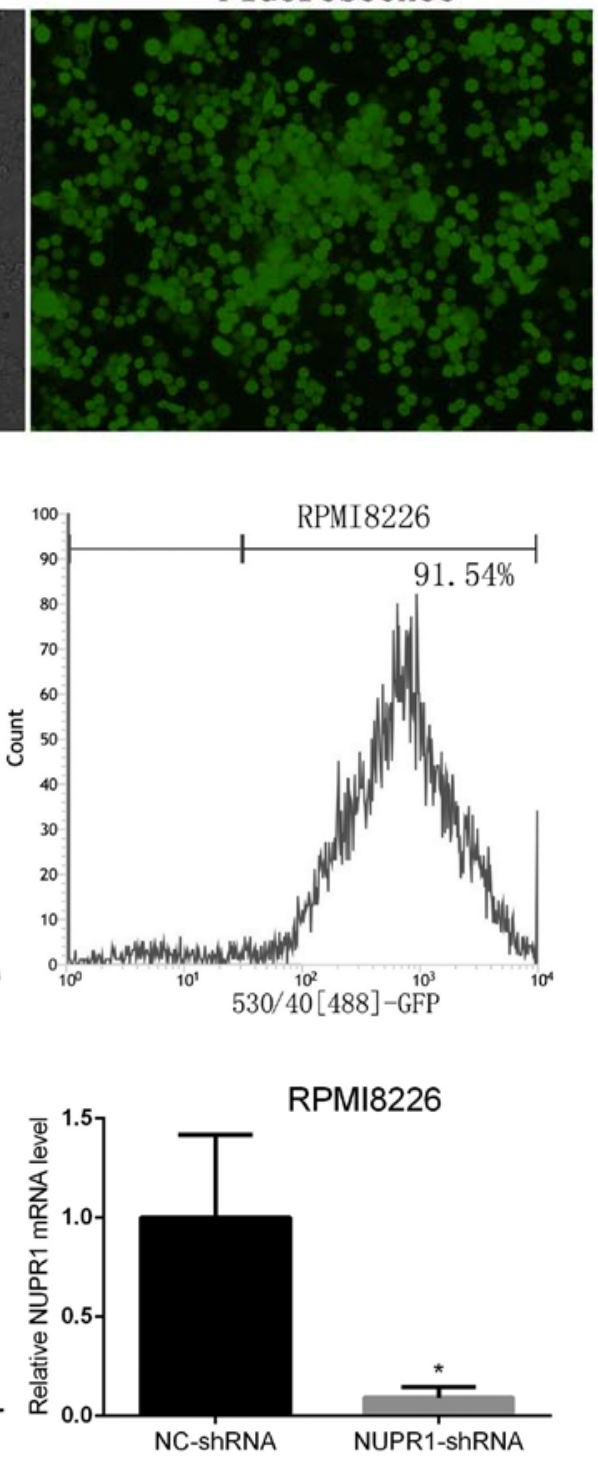

RPMI8226

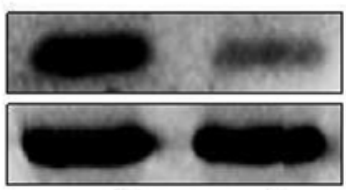

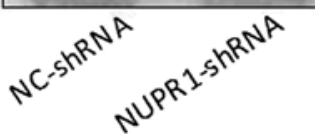

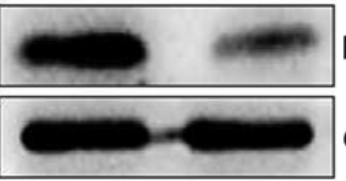

NUPR1 (17 kDa)

GAPDH (37 kDa)

Figure 2. Transfection of lentivirus for targeted knockdown of the expression of NUPR1. (A) Green fluorescent protein expressed in U266 and RPMI8226 cells transfected with NUPR1-shRNA (magnification, x200). (B) Transfection efficiency was measured by flow cytometry. (C) Reverse transcription-quantitative polymerase chain reaction and western blot analyses revealed the expression levels of NUPR1 in the NUPR1-shRNA group compared with the NC-shRNA group $\left(\right.$ P $\left.<0.05,{ }^{* *} \mathrm{P}<0.01\right)$. NUPR1, nuclear protein-1; shRNA, short hairpin RNA; NC, negative control. 

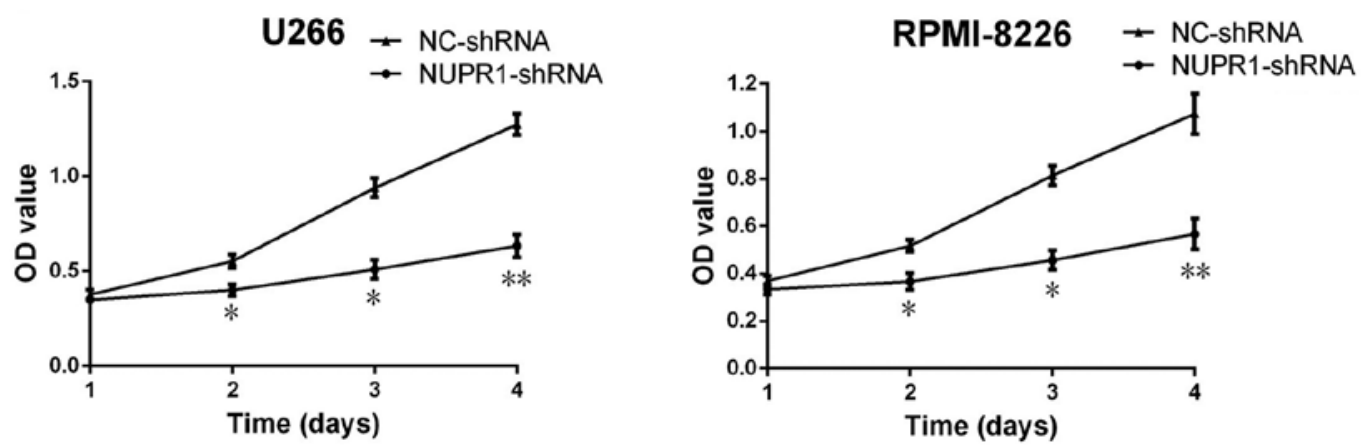

Figure 3. Proliferation of U266 and RPMI8226 cells was assessed using a Cell Counting Kit-8 assay. The viability of U266 and RPMI8226 cells was significantly inhibited in the NUPR1-shRNA group compared with that in the control group ( $\left.\mathrm{P}<0.05,{ }^{* *} \mathrm{P}<0.01\right)$. NUPR1, nuclear protein-1; shRNA, short hairpin RNA; NC, negative control.

evaluated following lentivirus infection for 5 days. The cells were resuspended and plated in 96-well plates at 2,000 cells per well. CCK-8 (10 $\mu$; Dojindo Molecular Technologies, Inc., Kumamoto, Japan) reagent was added to each well, and the plates were incubated at $37^{\circ} \mathrm{C}$ for $2 \mathrm{~h}$. Cell growth was estimated by measuring the absorbance at $450 \mathrm{~nm}$ with a microplate reader (M88; Thermo Fisher Scientific, Inc.) at the end of incubation.

Cell cycle analysis by fluorescence-activated cell sorting (FACS). The cells were collected and centrifuged at $1,500 \times \mathrm{g}$ at $22^{\circ} \mathrm{C}$ for $5 \mathrm{~min}$. The cells were then washed with PBS, and fixed with cold $70 \%$ ethanol at $4^{\circ} \mathrm{C}$ overnight. The cells were then stained with $50 \mu \mathrm{g} / \mathrm{ml}$ propidium iodide (PI) for $30 \mathrm{~min}$ at room temperature. Finally, each group of cells was determined using a FACScan cell sorter (BD Influx; BD Biosciences, Franklin Lakes, NJ, USA).

Assessment of apoptosis by flow cytometry. The cells were collected and stained with annexin V-APC to differentiate intact cells from apoptotic cells. All cells were washed with ice-cold PBS twice and incubated for $30 \mathrm{~min}$ in a binding buffer (1 mg/ml annexin V-APC), respectively. FACS analysis for annexin V-APC staining was performed by flow cytometry using CellQuest software version 4.02 (BD Biosciences).

Statistical analysis. The differences between groups were analyzed using Student's t-test or one-way analysis of variance using SPSS 22 software (IBM Corp., Armonk, NY, USA). Data are expressed as the mean \pm standard deviation. $\mathrm{P}<0.05$ was considered to indicate a statistically significant difference.

\section{Results}

Expression pattern of NUPR1 in human MM. The present study determined the mRNA expression of NUPR1 in U266 and RPMI8226 MM cell lines, and the specimens of patients with MM, with cells from normal human bone marrow as a control. As shown in Fig. 1, the MM cell lines and MM patient specimens showed higher expression levels of NUPR1, compared with those in the normal bone marrow cells $(\mathrm{P}<0.05)$. Therefore, a specific lentiviral vector was designed to knock down the NUPR1 gene and examine the role of the gene in the U266 and RPMI8226 MM cell lines.
Downregulation of the expression of NUPR1 in U266 and RPMI8226 cell lines following lentivirus transfection. To better understand the significance of the upregulation of NUPR 1 in the MM cell lines, the lentivirus expressing shRNA against NUPR1 was used for gene silencing in the U266 and RPMI8226 cells. The transfection efficiency of the cells was detected using a fluorescence microscope (Fig. 2A) and flow cytometry (Fig. 2B), the results of which showed that the transfection efficiency was $>80 \%$. The expression mRNA and protein levels of NUPR1 were determined by RT-qPCR and western blot analyses, respectively, following infection of the cells with the lentiviral vector (shCtrl or shNUPR1). The shNUPR1-transfected cells showed a significant reduction in mRNA and protein levels of NUPR1 when compared with the cells infected with the control lentivirus (Fig. 2C).

Downregulation of NUPR1 suppresses cell growth in U266 and RPMI8226 cells. To examine the potential function of NUPR1 in the growth of U266 and RPMI8226 cells, CCK-8 assays were used to analyze cell proliferation. Following a 3-day period, the proliferation of cells in the NUPR1-shRNA group was significantly suppressed compared with those in the negative control group (Fig. 3).

Analysis of cell cycle and apoptosis. Flow cytometry was performed to analyze apoptosis, as shown in Fig. 4A and B, the average apoptotic rates of the NUPR1-shRNA group and negative group U226 cells were $29.61 \pm 11.90$ and $6.18 \pm 1.13 \%$, and those of the RPMI8226 cells were $23.98 \pm 9.29$ and $8.11 \pm 2.75 \%$, respectively $(\mathrm{P}<0.05)$. This result revealed that knockdown of the expression of NUPR1 significantly promoted apoptosis of the U226 and RPMI8226 cells. FACS was used to examine the effect of NUPR1 knockdown on the cell cycle. As shown in Fig. 4C and D, compared with the negative group, the U266 and RPMI8226 cells in the NUPR1-shRNA group were arrested at the $\mathrm{G}_{0} / \mathrm{G}_{1}$ phase $(\mathrm{P}<0.01)$.

Knockdown of NUPR1 affects the expression of apoptotic and proliferation proteins in U266 and RPMI8226 cells. To investigate the molecular mechanism by which NUPR1 affects apoptosis and proliferation, the expression of relevant factors, including Bcl-2, cleaved caspase-3, -8 and -9, PTEN and PCNA, were examined in U266 and RPMI8226 cells with NUPR1 knockdown. The RT-qPCR and western blot analyses 
A

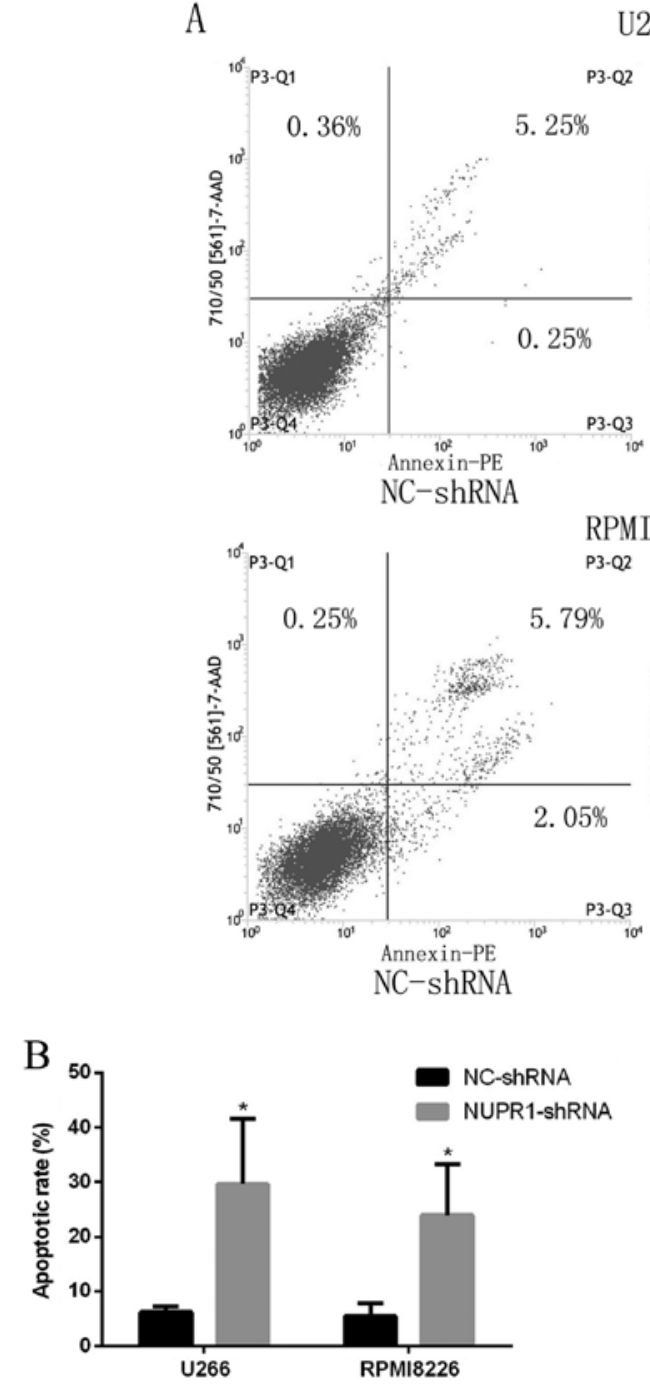

U266

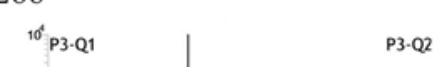

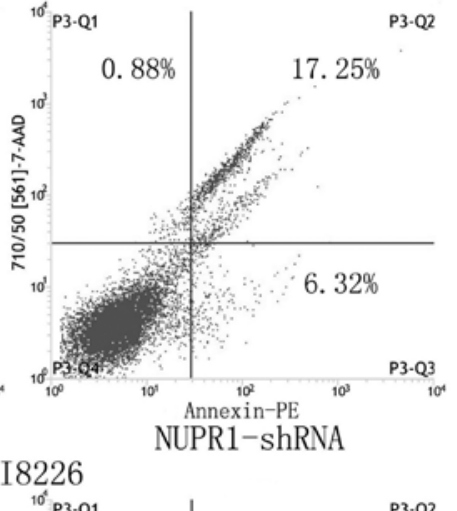

$\mathrm{D}$
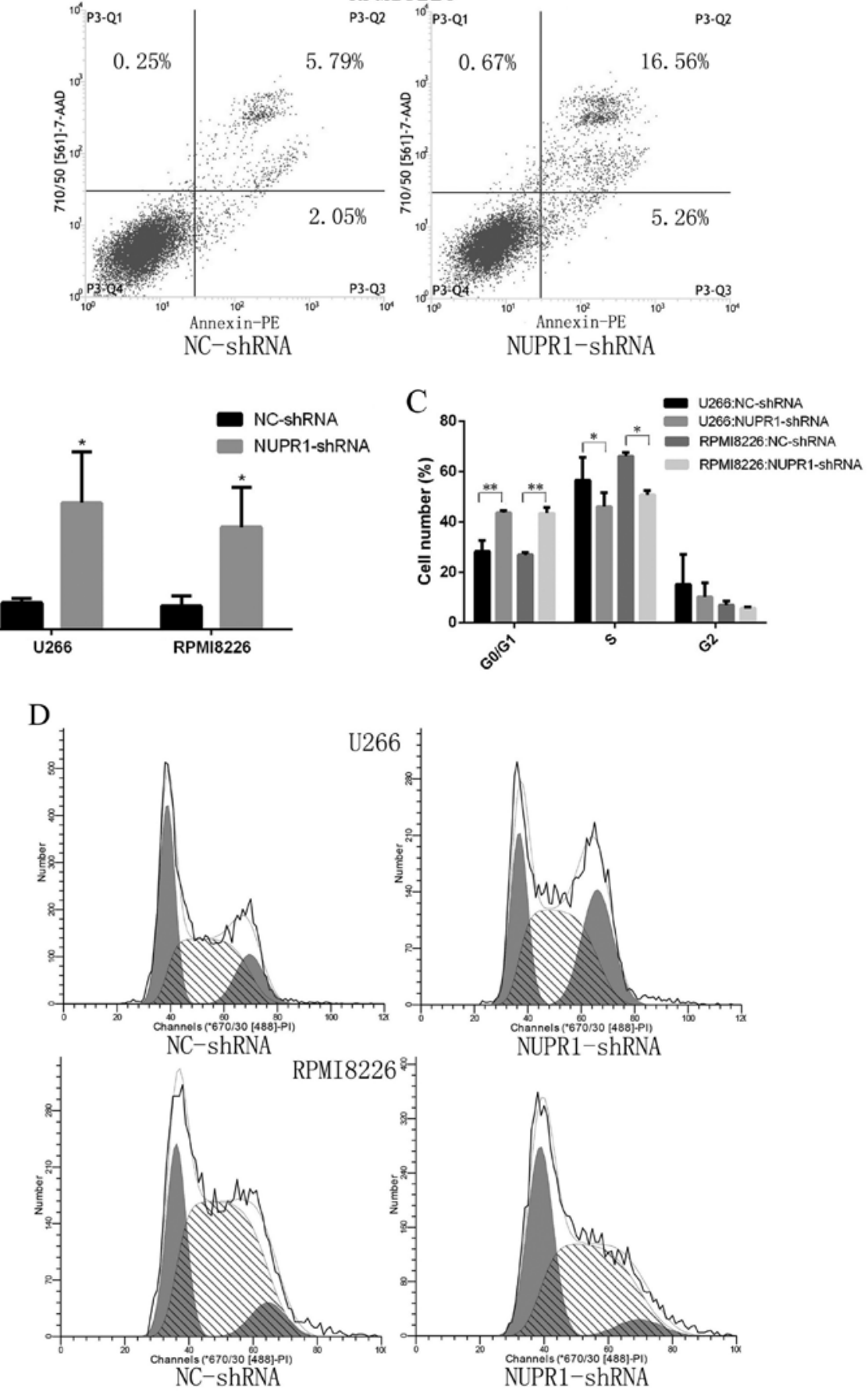

Figure 4. Effect of knockdown of the expression of NUPR1 on U266 and RPMI8226 cell apoptosis and cycle. (A) Apoptotic rate was evaluated by flow cytometry following transfection with lentiviral shRNA. (B) Analyzed results of apoptotic rates. (C) Analyzed results of cell cycle. ( $\left.\mathrm{P}<0.05,{ }^{* *} \mathrm{P}<0.01\right)$. (D) NUPR1 knockdown decreased the $(\mathrm{S}+\mathrm{G} 2) /(\mathrm{G} 1+\mathrm{S}+\mathrm{G} 2)$ proliferation index in the NUPR1-shRNA groups of U266 and RPMI8226 cells. NUPR1, nuclear protein-1; shRNA, short hairpin RNA; NC, negative control. 
A

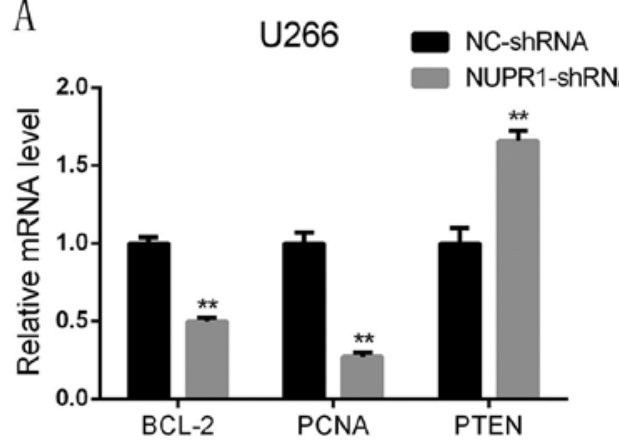

B

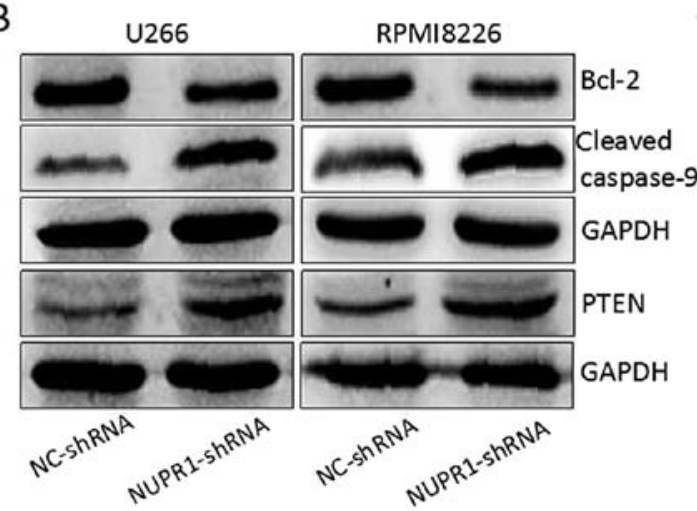

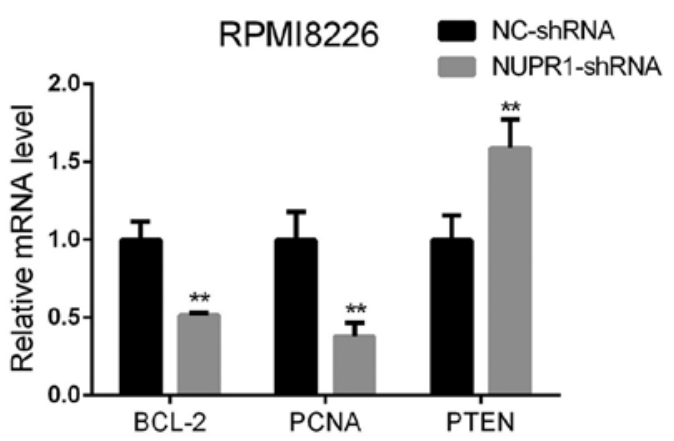

C

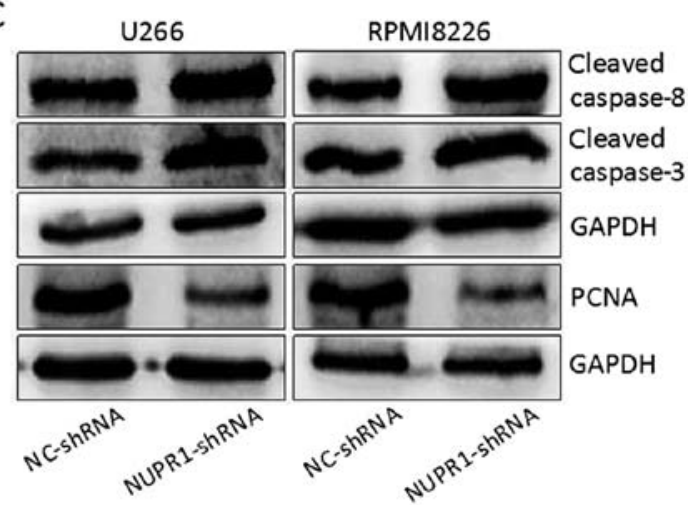

Figure 5. NUPR1 knockdown inhibits the expression of BCL-2 and PCNA, increases the expression of PTEN, and activates caspase-3, -8 and -9. (A) Reverse transcription-quantitative polymerase chain reaction analysis revealed that the mRNA expression levels of BCL-2 and PCNA decreased and the expression of PTEN increased in the NUPR1-shRNA group, compared with those in the NC group of U266 and RPMI18226 cells (** $<0.01)$. (B and C) Protein expression levels of PCNA, PTEN, BCL-2, and cleaved caspase-3, -9 and -8 were determined using a western blot assay. NUPR1, nuclear protein-1; PCNA, proliferating cell nuclear antigen; PTEN; phosphatase and tensin homolog; BCL-2, B-cell lymphoma 2; shRNA, short hairpin RNA; NC, negative control.

showed that the mRNA and protein levels of Bcl-2 and PCNA were significantly decreased in the NUPR1 shRNA-infected cells, whereas the expression level of PTEN was notably higher compared with that in the negative group. Western blot analysis revealed that the protein levels of cleaved caspase- $3,-8$ and -9 were higher in the U266 and RPMI8226 cells infected with the NUPR1-shRNA lentivirus, compared with those in the negative control group (Fig. 5A-C).

\section{Discussion}

NUPR1 was originally identified from the rat pancreas during the acute phase of pancreatitis (5). As a stress induced protein, it appears to be involved in a variety of stress-related functions and can produce different effects based on the physiological scenario. It is capable of promoting tumor growth and aggressiveness $(14,15)$, however, the same molecule appears to have tumor suppressive activity in prostate cancer (16), which depends on cancer cell type. This may be due to the different microenvironment affecting the activity of NUPR1. Previous studies have shown that NUPR1 affects the biological functions of tumor cells by regulating apoptosis and proliferation (17-19). NUPR1 modulates the apoptosis and proliferation of glioblastoma cells via caspase activation and the extracellular signal-regulated kinase signaling pathway (20). As an anti-apoptotic molecule, NUPR1 exerts an anti-apoptotic effect in breast cancer specimens and pancreatic cancer $(8,21,22)$.
However, whether and how NUPR1 affects MM cell viability remains to be fully elucidated, and few studies have been performed to determine the effects of NUPR1 on the behavior of MM cells in MM. In the present study, RT-qPCR was used to detect NUPR1 and it was found that the levels of NUPR1 in MM cell lines and specimens of patients with MM were significantly higher, compared with those in normal bone marrow cells. These results indicated that NUPR1 may be important in the progression of MM. The effects of NUPR1 on U266 and RPMI8226 MM cell line proliferation and apoptosis were also examined by infecting cells with an NUPR1-specific RNAi-expressing lentivirus. Apoptosis occurs in various physiological and pathological situations, and is involved in maintaining tissue homeostasis in multicellular organisms. Apoptosis may be initiated through different entry sites, including death receptors or mitochondria, which results in the activation of effector caspases $(23,24)$. Resistance to apoptosis is one of the hallmarks of human cancer, and promotes the development and progression of cancer (25). In addition, evasion from apoptosis represents one of the leading causes of failure of antileukemic therapy as numerous anticancer treatments act by triggering apoptosis in cancer cells (26). The present study indicated that the NUPR1-shRNA-infected cells exhibited reduced cell proliferation and increased apoptosis, and FACS analysis demonstrated that the NUPR1-shRNA-infected U266 and RPMI8226 cells were arrested at the $G_{0} / G_{1}$ phase, compared with the negative control group cells. 
Furthermore, the present study found that the knockdown of NUPR1 reduced the expression of PCNA and increased the expression of PTEN in U266 and RPMI8226 cells. PCNA is closely associated with cell DNA synthesis and repair, and promotes cell proliferation by increasing the synthesis of DNA polymerase (27). Following the knocking down of NUPR1, the gene and protein levels of PCNA were significantly downregulated, indicating that downregulating NUPR1 inhibited the proliferation activity of cells, possibly by affecting DNA synthesis and repair in MM cells. PTEN has been identified as a tumor suppressor, and mutation of this gene may lead to the development of several types of cancer. It has been reported that PTEN can regulate proliferation and apoptosis in different types of cancer (28-31). Studies have show that via the phosphoinositide 3-kinase (PI3K)/AKT signaling pathway, PTEN can regulate cell proliferation, and caspase-9 also regulated by Akt $(32,33)$. As the activation of phosphorylated Akt can lead to the decrease of Bcl-2 and the increase of cleaved caspase-3 (34), the present study examined the expression of Bcl-2, and cleaved caspase-3, -8 and -9 , which are general apoptosis-related proteins. It was found that the knockdown of NUPR1 inhibited the expression of Bcl-2 and promoted the expression of cleaved caspase- $3,-8$ and -9 , showing that inhibiting the expression of NUPR 1 may directly or indirectly lead to the downregulation of Bcl-2 and activation of caspase-3, -8 and -9 via the PTEN/PI3K/AKT signaling pathway, which is responsible for the inhibition of U266 and RPMI8226 cell growth in vitro. However, for specific signaling pathways of NUPR1 in MM cells and its in vivo mechanisms, further investigations are required in subsequent experiments. Only a preliminary judgment can be made on the expression level of NUPR1 in patients with MM. In subsequent experiments, an increase in the number of specimens is required to analyze the expression of NUPR1, and its association with patient outcomes and prognosis.

In conclusion, the present study showed that lentivirus-mediated NUPR1-shRNA (RNAi targeting NUPR1) significantly inhibited the proliferation of U266 and RPMI8226 MM cell lines, and induced apoptosis in vitro. NUPR1 may be associated with the induction of apoptosis by activating caspases and increasing the expression of PTEN in U266 and RPMI8226 cells. Further investigation of the functional role of NUPR1 may lead to an improved understanding of the molecular mechanism of MM. Drugs suppressing the effects of NUPR1 and inducing apoptosis may be a valid strategy for the treatment of MM.

\section{Acknowledgements}

The authors would like to thank Professor Jian Hou of The Second Military Medical University for providing the MM cell lines, and the Laboratory of Hematology of The First Affiliated Hospital of Chongqing Medical University for providing specimens. The authors would also like to thank Lixue Chen and Xiaojuan Deng (Experimental Research Center of the First Affiliated Hospital of Chongqing Medical University) for their technical assistance.

\section{Funding}

No funding was received.

\section{Availability of data and materials}

The datasets used during the present study are available from the corresponding author upon reasonable request.

\section{Authors' contributions}

$\mathrm{CZ}$ and JC conceived and designed the study. CZ, XL, AL and $\mathrm{BY}$ performed the experiments. $\mathrm{CZ}, \mathrm{XP}$ and $\mathrm{XH}$ wrote the manuscript. $\mathrm{CZ}, \mathrm{XP}, \mathrm{XH}$ and JC reviewed and edited the manuscript. All authors read and approved the manuscript and agree to be accountable for all aspects of the research in ensuring that the accuracy or integrity of any part of the work are appropriately investigated and resolved.

\section{Ethics approval and consent to participate}

All specimens were obtained in accordance with the Research Ethics Board of the First Affiliated Hospital of Chongqing Medical University. Informed consent was provided.

\section{Patient consent for publication}

Not applicable.

\section{Competing interests}

The authors declare that they have no competing interests.

\section{References}

1. Mohamed AN, Bentley G, Bonnett ML, Zonder J and Al-Katib A: Chromosome aberrations in a series of 120 multiple myeloma cases with abnormal karyotypes. Am J Hematol 82: 1080-1087, 2007.

2. Dupéré-Richer D and Licht JD: Epigenetic regulatory mutations and epigenetic therapy for multiple myeloma. Curr Opin Hematol 24: 336-344, 2017.

3. Manier S, Salem KZ, Park J, Landau DA, Getz G and Ghobrial IM: Genomic complexity of multiple myeloma and its clinical implications. Nat Rev Clin Oncol 14: 100-113, 2017.

4. Mallo GV, Fiedler F, Calvo EL, Ortiz EM, Vasseur S, Keim V, Morisset J and Lovanna JL: Cloning and expression of the rat $\mathrm{p} 8$ cDNA, a new gene activated in pancreas during the acute phase of pancreatitis, pancreatic development, and regeneration, and which promotes cellular growth. J Biol Chem 272: 32360-32369, 1997.

5. Cano CE, Hamidi T, Sandi MJ and Iovanna JL: Nupr1: The Swiss-knife of cancer. J Cell Physiol 226: 1439-1443, 2011.

6. Sandi MJ, Hamidi T, Malicet C, Cano C, Loncle C, Pierres A, Dagorn JC and Lovanna JL: p8 expression controls pancreatic cancer cell migration, invasion, adhesion, and tumorigenesis. J Cell Physiol 226: 3442-3451, 2011.

7. Emma MR, Iovanna JL, Bachvarov D, Puleio R, Loria GR, Auqello G, Candido S, Libra M, Gulino A, Cancila V, et al: NUPR1, a new target in liver cancer: Implication in controlling cell growth, migration, invasion and sorafenib resistance. Cell Death Dis 7: e2269, 2016.

8. Veerla S, Panagopoulos I, Jin Y, Lindgren D and Höglund M: Promoter analysis of epigenetically controlled genes in bladder cancer. Genes Chromosomes Cancer 47: 368-378, 2008.

9. Ito Y, Yoshida H, Motoo Y, Lovanna JL, Nakamura Y, Kakudo K, Uruno T, Takamura Y, Miya A, Noquchi S, et al: Expression of p8 protein in breast carcinoma; an inverse relationship with apoptosis. Anticancer Res 25: 833-837, 2005.

10. Di Martino MT, Guzzi PH, Caracciolo D, Aqnelli L, Neri A, Walker BA, Morqan GJ, Cannataro M, Tassone P and Taqliaferri P: Integrated analysis of microRNAs, transcription factors and target genes expression discloses a specific molecular architecture of hyperdiploid multiple myeloma. Oncotarget 6: 19132-19147, 2015 
11. Palumbo A, Avet-Loiseau H, Oliva S, Lokhorst HM, Goldschmidt H, Rosinol L, Richardson P, Caltagirone S, Lahuerta JJ, Facon T, et al: Revised international staging system for multiple myeloma: A report from international myeloma working group. J Clin Oncol 33: 2863-2869, 2015.

12. Scherr M and Eder M: Gene transfer into hematopoietic stem cells using lentiviral vectors. Curr Gene Ther 2: 45-55, 2002.

13. Livak KJ and Schmittgen TD: Analysis of relative gene expression data using real-time quantitative PCR and the $2^{-\triangle \Delta C T}$ method. Methods 25: 402-408, 2001.

14. Guo X, Wang W, Hu J, Feng K, Pan Y, Zhang L and Feng L: Lentivirus-mediated RNAi knockdown of NUPR1 inhibits human nonsmall cell lung cancer growth in vitro and in vivo. Anat Rec 295: 2114-2121, 2012.

15. Pedrola N, Devis L, Llauradó M, Campoy I, Martinez-Garcia E, Garcia M, Muinelo-Romay L, Alonso-Alconada L, Abal M, Alameda F, et al: Nidogen 1 and nuclear protein 1: Novel targets of ETV5 transcription factor involved in endometrial cancer invasion. Clin Exp Metastasis 32: 467-478, 2015.

16. Jiang WG, Davies G, Martin TA, Kynaston H, Mason MD and Fodstad O: Com-1/p8 acts as a putative tumour suppressor in prostate cancer. Int J Mol Med 18: 981-986, 2006.

17. Kong DK, Georgescu SP, Cano C, Aronovitz MJ, Lovanna JL, Patten RD, Kyriakis JM and Goruppi S: Deficiency of the transcriptional regulator $\mathrm{p} 8$ results in increased autophagy and apoptosis, and causes impaired heart function. Mol Biol Cell 21: 1335-1349, 2010.

18. Goruppi S and Iovanna JL: Stress-inducible protein $\mathrm{p} 8$ is involved in several physiological and pathological processes. J Biol Chem 285: 1577-1581, 2010.

19. Li J, Ren S, Liu Y, Lian Z, Dong B, Yao Y and Xu Y: Knockdown of NUPR1 inhibits the proliferation of glioblastoma cells via ERK1/2, p38 MAPK and caspase-3. J Neurooncol 132: 15-26, 2017.

20. Lorente M, Carracedo A, Torres S, Natali F, Eqia A, Hernández-Tiedra S, Salazar M, Blázquez C, Guzman M and Velasco G: Amphiregulin is a factor for resistance of glioma cells to cannabinoid-induced apoptosis. Glia 57: 1374-1385, 2009.

21. Su SB, Motoo Y, Iovanna JL, Berthézène P, Xie MJ, Mouri H, Ohtsubo K, Matsubara F and Sawabu N: Overexpression of p8 is inversely correlated with apoptosis in pancreatic cancer. Clin Cancer Res 7: 1320-1324, 2001.

22. Malicet C, Giroux V, Vasseur S, Dagorn JC, Neira JL and Iovanna JL: Regulation of apoptosis by the p8/prothymosin alpha complex. Proc Natl Acad Sci USA 103: 2671-2676, 2006.
23. Fulda S and Debatin KM: Extrinsic versus intrinsic apoptosis pathways in anticancer chemotherapy. Oncogene 25: 4798-4811, 2006.

24. Hengartner MO: The biochemistry of apoptosis. Nature 407: $770-776,2000$

25. Hanahan D and Weinberg RA: Hallmarks of cancer: The next generation. Cell 144: 646-674, 2011.

26. Reed JC and Pellecchia M: Apoptosis-based therapies for hematologic malignancies. Blood 106: 408-418, 2005.

27. Mahler M, Miyachi K, Peebles C and Fritzler MJ: The clinical significance of autoantibodies to the proliferating cell nuclear antigen (PCNA). Autoimmun Rev 11: 771-775, 2012.

28. Gregorian C, Nakashima J, Dry SM, Nghiemphu PL, Smith KB, Ao Y, Dang J, Lawson G, Mellinghoff IK, Mischel PS, et al: PTEN dosage is essential for neurofibroma development and malignant transformation. Proc Natl Acad Sci USA 106: 19479-19484, 2009.

29. Song L, Liu S, Zhang L, Yao H, Gao F, Xu D and Li Q: MiR-21 modulates radiosensitivity of cervical cancer through inhibiting autophagy via the PTEN/Akt/HIF-1alpha feedback loop and the Akt-mTOR signaling pathway. Tumour Biol 37: 12161-12168, 2016.

30. Wang DD, Yang SJ, Chen X, Shen HY, Luo LJ, Zhang XH, Zhong SL, Zhao JH and Tang JH: miR-222 induces Adriamycin resistance in breast cancer through PTEN/Akt/p27 kipl pathway. Tumour Biol 37: 15315-15324, 2016.

31. Zhu DY, Li XN, Qi Y, Liu DL, Yang Y, Zhao J, Zhang CY, $\mathrm{Wu} \mathrm{K}$ and Zhao S: MiR-454 promotes the progression of human non-small cell lung cancer and directly targets PTEN. Biomed Pharmacother 81: 79-85, 2016.

32. Xiaozhou L, Xing Z, Haidong X, Zhiwei H, Xin S and Sujia W: SLC34A2 regulates the proliferation, migration, and invasion of human osteosarcoma cells through PTEN/PI3K/AKT signaling. DNA Cell Biol 36: 775-780, 2017.

33. Chappell WH, Steelman LS, Long JM, Kempf RC, Abrams SL, Franklin RA, Bäsecke J, Stivala F, Donia M, Fagone P, et al: Ras/Raf/MEK/ERK and PI3K/PTEN/Akt/mTOR inhibitors: Rationale and importance to inhibiting these pathways in human health. Oncotarget 2: 135-164, 2011.

34. Yang F, Shi L, Liang T, Ji L, Zhang G, Shen Y, Zhu F and Xu L: Anti-tumor effect of evodiamine by inducing Akt-mediated apoptosis in hepatocellular carcinoma. Biochem Biophys Res Commun 485: 54-61, 2017. 